Diamond detectors for fast neutron measurements at pulsed spallation sources

This article has been downloaded from IOPscience. Please scroll down to see the full text article.

2012 JINST 7 C05015

(http://iopscience.iop.org/1748-0221/7/05/C05015)

View the table of contents for this issue, or go to the journal homepage for more

Download details:

IP Address: 115.42.147.242

The article was downloaded on 25/06/2012 at 17:20

Please note that terms and conditions apply. 


\title{
Diamond detectors for fast neutron measurements at pulsed spallation sources
}

\author{
M. Rebai, ${ }^{a}{ }^{1}$ L. Giacomelli, ${ }^{a}$ C. Andreani, ${ }^{b}$ A. Fazzi, ${ }^{c}$ C.D. Frost,${ }^{d}$ E. Perelli Cippo, ${ }^{a}$ \\ A. Pietropaolo, ${ }^{a}$ N. Rhodes, ${ }^{d}$ M. Tardocchi, ${ }^{e}$ E. Schooneveld ${ }^{d}$ and G. Gorini ${ }^{a, b, c, d, e}$ \\ ${ }^{a}$ University of Milano Bicocca, \\ Piazza della Scienza 3, 20126 Milano, Italy \\ ${ }^{b}$ University of Roma Tor Vergata, Dipartimento di Fisica and Centro NAST, \\ Via della Ricerca Scientifica 1, 00133 Roma, Italy \\ ${ }^{c}$ Energy Department, Politecnico of Milano, \\ Via Ponzio 32, Milano, Italy \\ ${ }^{d}$ STFC, Rutherford Appleton Laboratory, \\ Didcot, OX11 0QX, U.K. \\ ${ }^{e} I F P-C N R$, \\ Via Cozzi 53, 20125 Milano, Italy \\ E-mail: marica.rebai@mib.infn.it
}

ABSTRACT: The performance of a single crystal diamond $\left(4.7 \times 4.7 \times 0.5 \mathrm{~mm}^{3}\right.$ active volume $)$ detector was tested in the ISIS pulsed neutron beam using biparametric (time of flight and pulse height) data acquisition. Three characteristic regions in the biparametric spectra are observed: i) low pulse height events with very short time of flight induced by $\gamma$-rays; ii) low pulse height events at longer flight times (i.e. neutron energies $E_{n}>3.5-6 \mathrm{MeV}$ ), possibly due to neutron elastic scattering off ${ }^{12} \mathrm{C}$; iii) events with large pulse height and flight times corresponding to $E_{n}>6 \mathrm{MeV}$ mainly due to inelastic reactions such as ${ }^{12} \mathrm{C}(\mathrm{n}, \alpha){ }^{9} \mathrm{Be}$ and ${ }^{12} \mathrm{C}\left(\mathrm{n}, \mathrm{n}^{\prime}\right) 3 \alpha$. The potential use of this detector is discussed in relation to the ChipIr neutron beam line for fast neutron irradiation of electronic components at the ISIS spallation source.

KEYWORDS: Solid state detectors; Instrumentation for neutron sources; Neutron sources; Neutron detectors (cold, thermal, fast neutrons)

\footnotetext{
${ }^{1}$ Corresponding author.
} 


\section{Contents}

1 Introduction 1

2 Experimental 2

3 Results 4

$\begin{array}{lll}4 & \text { Biparametric analysis } & 7\end{array}$

5 Beam profile measurements $\quad 12$

6 Discussion on the expected neutron spectra 13

$\begin{array}{lll}7 & \text { Conclusions } & 14\end{array}$

\section{Introduction}

Electronic devices operating at sea level and on aircrafts are exposed to various types of cosmic ray radiation such as protons, neutrons and muons. The so called Single Event Effects (SEEs) [1, 2] in digital electronics - ranging from temporary loss of data (soft errors) to catastrophic failures (hard errors) - are mainly due to neutrons of energy $E_{n}>1 \mathrm{MeV}$. Fault-tolerant design techniques must be used, and extensive analysis is needed in order to assess the robustness of devices and systems. Experiments with atmospheric neutrons at different altitudes can be carried out, but require very long periods of data acquisition. Neutron sources can provide much higher fluxes thus allowing for accelerated irradiation experiments. Dedicated beam lines are available at facilities worldwide, such as LANSCE (US) [3], TRIUMF (Canada) [4] and ANITA (Sweden) [5]. At the ISIS spallation neutron source (Didcot, U.K.) a dedicated beam line (ChipIr) is under construction [6]. Suitable neutron beam monitors and spectrometers are needed for measurements of the ChipIr neutron flux in the multi-MeV energy range. A variety of fast neutron detection methods can be considered; some are already in use at existing irradiation facilities such as the Thin Film Breakdown Counter (TFBC) at ANITA [7]. Detailed investigations of the time structure of the ISIS neutron pulse performed with TFBCs on the VESUVIO beamline at ISIS [8] have shown that it can be used to extract the main features of the energy spectrum of fast neutrons with $E_{n}>1 \mathrm{MeV}$ by using the time of flight method. The use of TFBCs for quantitative spectroscopy is made possible by accurate knowledge of fission cross sections such as ${ }^{238} \mathrm{U}(\mathrm{n}, \mathrm{f}),{ }^{235} \mathrm{U}(\mathrm{n}, \mathrm{f}),{ }^{209} \mathrm{Bi}(\mathrm{n}, \mathrm{f})$.

The detector for fast neutron measurements at ISIS investigated in this paper is a single-crystal diamond detector (SDD). Diamond detectors, thanks to the high mobility of charge carriers, feature a very fast response time, in the order of few ns. This property, combined with the radiation hardness of the detection material, make it an attractive choice for measurements of high fluxes of radiation including neutrons. The applications of SDDs are rapidly growing. They range from UV 
detection for astrophysics and plasma physics, to minimum ionizing particle detection in particle physics experiments, $X$ - and $\gamma$-ray detection for radiology and radiotherapy [9], and proton beam sensors [10]. SDD applications for fast neutron measurements include neutron emission monitors in nuclear fusion experiments [11] and neutron spectrometers [12]. The performance of these detectors is generally very good although transient polarization effects have been observed in some cases [13]. The detectors used in the measurements presented here are from Diamond Detectors Ltd. (DDL) [14]. The response of this type of detector to monoenergetic neutrons in the range up to $20 \mathrm{MeV}$ was recently investigated in detail [15]. It features sharp peaks in the pulse height spectrum due to reactions with only charged particles in the exit channel (the two body reactions ${ }^{12} \mathrm{C}(\mathrm{n}, \alpha){ }^{9} \mathrm{Be},{ }^{12} \mathrm{C}(\mathrm{n}, \mathrm{p}){ }^{11} \mathrm{~B},{ }^{12} \mathrm{C}(\mathrm{n}, \mathrm{d}){ }^{10} \mathrm{~B}$ and $\left.{ }^{13} \mathrm{C}(\mathrm{n}, \alpha){ }^{10} \mathrm{Be}\right)$ and wide distributions with characteristic edges when a neutron is present in the exit channel $\left({ }^{12} \mathrm{C}\left(\mathrm{n}, \mathrm{n}^{\prime}\right){ }^{12} \mathrm{C},{ }^{12} \mathrm{C}\left(\mathrm{n}, \mathrm{n}^{\prime}\right) 3 \alpha\right)$. The complexity of the detector response does not prevent quantitative spectroscopy applications in cases when the neutron spectrum is nearly monoenergetic such as deuterium-tritium fusion [16]. In the case of neutrons from spallation sources the quantitative analysis of the pulse height spectrum would require accurate knowledge of the detector response over a much broader energy range, which is a demanding task - though less formidable than determining the energy-dependent cross sections of the many reaction channels involved.

Eventually the best approach for using SDDs at a pulsed spallation source like ISIS may be to combine pulse height analysis (as done in ordinary spectroscopy) with time of flight analysis (as done. e.g. with TFBCs). In principle time of flight analysis and pulse height analysis provide the same information on the neutron energy spectrum with limitations due to e.g. the fine time structure of the ISIS pulsed beam (the time spread of the proton pulse is not negligible) or the pulse height energy resolution of the SDD. By combining both analyses in one biparametric measurement one should in principle be able to overcome some of the limitations intrinsic in the use of time of flight analysis or pulse height analysis independently.

A first step towards the use of SDDs at ISIS is therefore to demonstrate reliable operation of the SDD detector in biparametric mode of operation in a high neutron flux. Early tests of diamond detectors were previously performed at ISIS and reported in [17-22]. These tests were performed at rather low neutron detection rates using custom made fine quality diamonds of small active volume. In the tests presented here, two larger SDDs were coupled to a fast waveform digitizer from CAEN [23] suitable for off-line biparametric (time of flight and pulse height) analysis. Goals of the tests were a general understanding of the biparametric spectrum and the search for possible polarization effects affecting the detector response stability over a time period of a few days. The detectors were also used to measure the neutron flux profile across the fast neutron beam.

\section{Experimental}

The measurements were performed with two SDD detectors placed in the neutron beam of the VESUVIO [24] beam line at a flight distance of about $L=12.5 \mathrm{~m}$ from the neutron source. At ISIS, neutrons are produced by a $800 \mathrm{MeV}$ proton beam with a double bunch fine structure and a repetition frequency of $50 \mathrm{~Hz}$. The two proton bunches are about $70 \mathrm{~ns}$ wide (FWHM) and $322 \mathrm{~ns}$ apart. The proton beam delivers an average current of $180 \mu \mathrm{A}$ on a Ta-W target yielding about 15-20 neutrons per incident proton. The VESUVIO neutron spectrum predicted by MCNP simulations is 


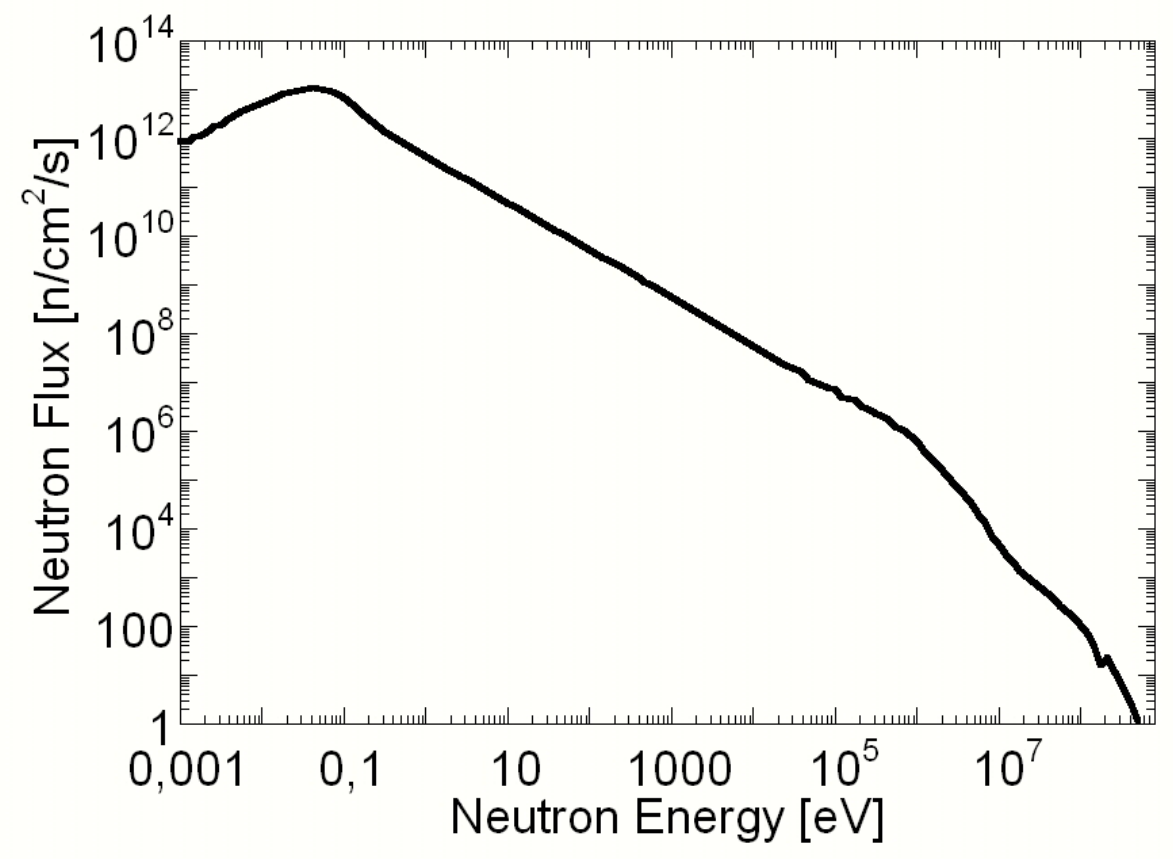

Figure 1. VESUVIO neutron spectrum.

shown in figure 1 . Neutrons coming from the spallation target are partially moderated by a $300 \mathrm{~K}$ water moderator, thus the spectrum is peaked at about $30 \mathrm{meV}$ and is known to decrease roughly as $1 / E^{\alpha}$, with $\alpha \approx 0.9$, in the epithermal energy region (above $0.5 \mathrm{eV}$ ). The undermoderation of neutrons results in the presence of an intense flux of neutrons above $1 \mathrm{MeV}$. The maximum neutron energy achievable is equal to the proton beam energy, i.e. $800 \mathrm{MeV}$.

Two diamond detectors from different production batches and featuring aluminium (Al-SDD) or gold (Au-SDD) as contact materials were placed in the neutron beam for about three days. The nominal active volume of the two detectors is identical being $4.7 \times 4.7 \mathrm{~mm}^{2}$ area and $0.5 \mathrm{~mm}$ thickness. The electrical contacts have $4.5 \mathrm{~mm}$ diameter. Both detectors were mounted on a movable support that was used to move the SDDs remotely in the horizontal direction during some of the measurements.

Two commercial fast preamplifiers, DBA III and DBA IV [25] were used to preserve the fast response of the SDDs. Both preamplifiers have a shaping time of about $10 \mathrm{~ns}$ and a broad bandwidth in the range $3 \mathrm{MHz}-3 \mathrm{GHz}$. The gain can be remotely controlled but was fixed in these experiments to the maximum available gain; i.e. $38 \mathrm{~dB}$ and $46 \mathrm{~dB}$ for the DBAIII and the DBA IV, respectively. An additional custom wideband amplifier (gain=4) was used in order to boost the amplitude of the signal from the DBAIII preamplifier. The signals from the two detectors were fed into the CAEN waveform digitizer model DT5751 [23]. This is a four channel desktop digitizer with a sampling frequency of $1 \mathrm{GHz}$, input range $0-1 \mathrm{~V}$ and 10 bit resolution. The waveforms from both SDDs were stored in coincidence with a reference signal generated by the proton extraction from the synchrotron. For each ISIS pulse a waveform of $2500 \mathrm{~ns}$ was stored; all time values were synchronised during the analysis relative to the arrival time of the photons at the detector. The latter is set so that the peak in time of the photon signal occurs at $L / c=42 \mathrm{~ns}$. 


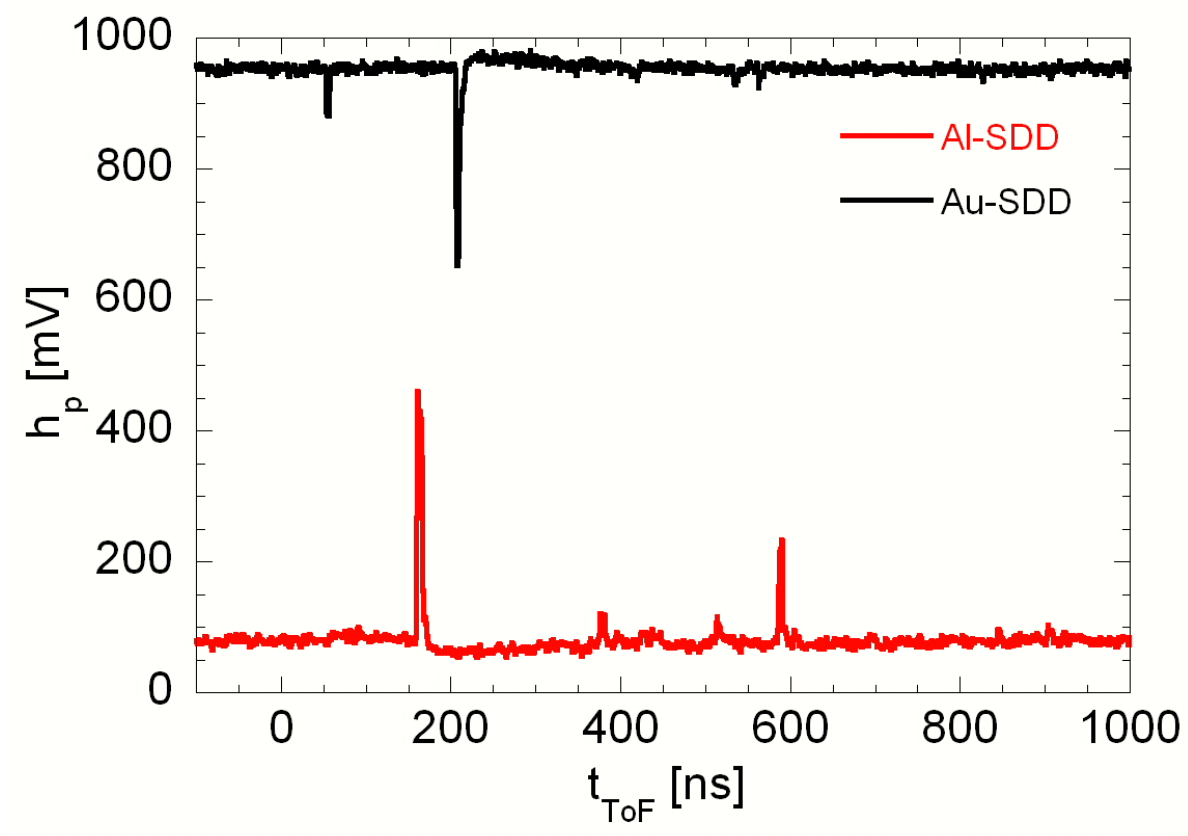

Figure 2. Data from the recorded waveform for the Au-SDD (black) and for the Al-SDD (red). The full waveform is $2500 \mathrm{~ns}$ long.

\section{Results}

An example of recorded waveforms from the two detectors is shown in figure 2. The different polarity is due to the use of the two different preamplifiers (DBAIII is inverting, DBAIV noninverting). Typically many narrow pulses (each about $10 \mathrm{~ns}$ long) were recorded in every $2500 \mathrm{~ns}$ long waveform. Several pulses can be recognised in figure 2 with pulse height $\left(h_{p}\right)$ values ranging from noise level (rms $=10 \mathrm{mV}$ for the DBAIII, and $6 \mathrm{mV}$ for the DBAIV) up to hundreds of $\mathrm{mV}$.

A dedicated Python [26] code was used for off-line analysis to extract information on pulse height $h_{p}$, amplitude $A$ (i.e. the area under the pulses in figure 2 ) and time of flight $\left(t_{T o F}\right)$ from the measured waveforms. The analysis is based on identification of all data points exceeding a threshold value (usually $20 \mathrm{mV}$ ) above the average offset level. These are tagged as events and $N=18$ data points around the pulse height maximum are used for each event to determine the pulse amplitude. The baseline is determined for each event from the 6 data points following the pulse and subtracted before computing the pulse amplitude. The time at maximum pulse height is used as $t_{T o F}$ value. It is important to note that the amplification and digitization approach used here was optimised for speed rather than pulse height resolution; hence the pulse height resolution is modest. To reduce the uncertainties the integral pulse height or amplitude $A$ was obtained from the sum of the $N$ data points for each event and used as a measure of the energy deposited in the SDD.

Energy calibration of the Au-SDD coupled to DBAIV was performed in the laboratory using a ${ }^{241} \mathrm{Am} \alpha$ source in air. The calibration results are shown in figure 3. The peak position is $665.5 \mathrm{mVns}$ and the FWHM is $96 \mathrm{mVns}$ or $14.4 \%$. This is a large FWHM compared to usual performance of these detectors and is due to the data digitization and analysis used. The energy of an $\alpha$ particle from ${ }^{241} \mathrm{Am}$ is about $5.5 \mathrm{MeV}$ but is reduced to $5.0 \mathrm{MeV}$ when the $\alpha$ article reaches 


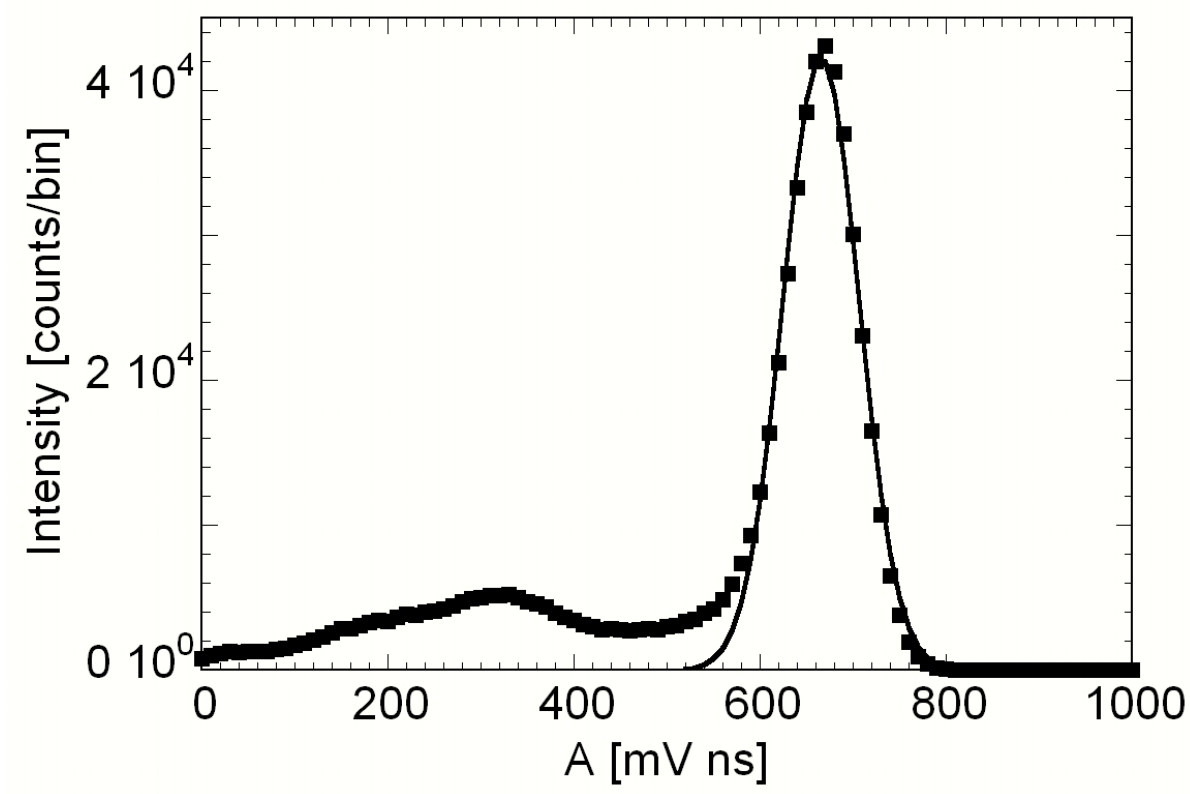

Figure 3. Calibration peak for the Au-SDD with ${ }^{241}$ Am source. The line is a Gaussian fit to the data. The Gaussian parameters are height $\mathrm{I}=4.5 \bullet 10^{4}$, FWHM= $96 \mathrm{mVns}$, position $\mathrm{A}=665.5 \mathrm{mVns}$.

the detector after crossing $5.7 \mathrm{~mm}$ of air. A calibration coefficient of $7.50 \cdot 10^{-3} \mathrm{MeV} /(\mathrm{mVns})$ that was thus determined and used to convert $A$ values to equivalent deposited energy $E_{d}$. The error in the calibration coefficient is mainly systematic and estimated to be $\approx 15 \%$.

A second energy calibration was performed during the experiments at ISIS for both detectors. Due to the lack of available calibration $\alpha$-particle sources at the measurement location, the $\alpha$-decay of a natural uranium foil was used as a calibration reference (thin uranium foils are in regular use at the VESUVIO beam line). The quality of the measured $\alpha$-particle spectra is poor but sufficient to provide a relative calibration for the two detectors that is consistent with the nominal amplification settings of the two detectors. For the Al-SDD we get a calibration coefficient of $5.04 \cdot 10^{-3} \mathrm{MeV} /(\mathrm{mVns})$.

The relation between measured pulse height $h_{p}$ and integral amplitude $A$ is shown by the scatter plot of figure 4 which includes a subset of all data collected with the Au-SDD and Al-SDD placed in the VESUVIO neutron beam. The data extend up to the maximum $h_{p}$ values allowed by the digitizer input (including some offset). A very small fraction of the events $(\approx 0.02 \%)$ saturates the digitizer input for the Au-SDD and was rejected from the analysis. The fraction of saturated events is larger for the Al-SDD $(\approx 0.09 \%)$. This suggests that the Al-SDD saturates earlier because of the amplification settings. In both cases the data show a large scatter around an average linear relation $A=\tau_{p}$ with $\tau=4 \mathfrak{u} \mathfrak{y} \mathfrak{y s}$ and $5.54 \mathrm{~ns}$ for the Au-SDD and Al-SDD, respectively. This is consistent with the use of a second amplification stage for Al-SDD slightly broadening the pulse shape. Taking into account the energy calibration of the detectors a pulse height value of $h_{p}=1 \mathrm{~V}$ translates to an energy of $32.4 \mathrm{MeV}$ and $28 \mathrm{MeV}$ for the Au-SDD and Al-SDD, respectively. As far as the maximum deposited energy is concerned, figure 4 gives a general indication of the trend of the signals' main parameters, i.e. area and pulse height. The linear fit represent the average value of 


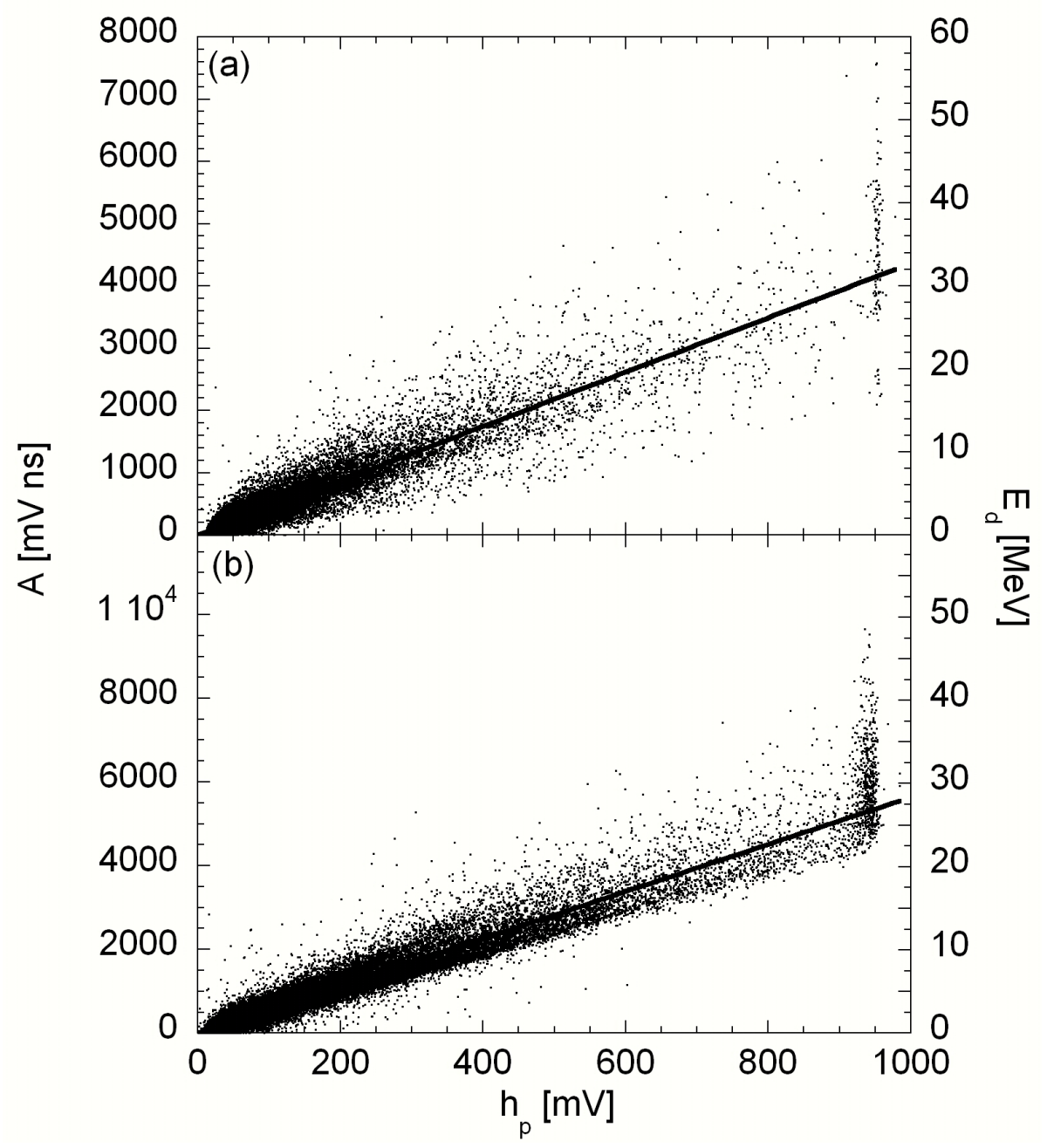

Figure 4. Scatter plot of $A$ vs $h_{p}$ for a subset of the data collected with the Au-SDD placed in the VESUVIO neutron beam. The line is a linear best fit to the data. (b) same as (a) but for the Al-SDD. The total number of data points is $2.9 \bullet 10^{5}$ in (a) and $7.4 \bullet 10^{5}$ in (b).

the deposited energy. The off line events in the high- $\mathrm{E}_{d}$ region, excluding the saturated signals, are genuine events. Indeed, these are due to ${ }^{-12} \mathrm{C}$ inelastic reactions above (at least) $10 \mathrm{MeV}$ incident neutron energy. Where above this energy the combined effect of low neutron flux and fast signal processing makes pile up almost unlikely. The threshold pulse height of $h_{p}=20 \mathrm{mV}$ translates to an energy of $0.65 \mathrm{MeV}$ and $0.56 \mathrm{MeV}$ for the Au-SDD and Al-SDD, respectively. These values set the maximum and minimum deposited energies in our measurements. Note that neither limit is sharp. For instance the threshold $h_{p}>20 \mathrm{mV}$ does not correspond to a sharp cut in the events plane in figure 4 . This is because the threshold is relative to an average offset whereas the actual offset subtracted to each event is different. The upper energy limit is more clearly recognizable in figure 4 due to the cluster of events with saturated pulse height.

By counting the pulses above a certain $h_{p}$ threshold it was possible to test the detector stability. The neutron count rate for $h_{p}>150 \mathrm{mV}$ (i.e. $E_{d}>4.19 \mathrm{MeV}$ ) was found to be stable at the percent 
level for a period of 30 hours using the Al-SDD. The average count rate was 3.5 count/s. Since most of the events occur within $600 \mathrm{~ns}$ (see below) of the proton interaction with the target, the peak count rate can be estimated as $\left(50 \times 6 \cdot 10^{-7}\right)^{-1}$ time the average count rate, or $\approx 100 \mathrm{kHz}$. The total count rate including events of lower deposited energy is much higher and exceeds $1 \mathrm{MHz}$ for events with $h_{p}>20 \mathrm{mV}$. This suggests that the fast signal amplification and digitization is essential for successful operation of an SDD in the ISIS environment. The total count rate of the Au-SDD and Al-SDD includes a large contribution from events of low pulse height and depends strongly on the $h_{p}$ threshold value and on the way the data are processed. For this reason the total count rate varies by a factor 2.53 between the Au-SDD and Al-SDD even if their response at high $E_{d}$ is similar (see below).

Figure 5 shows the biparametric (ToF- deposited energy, $\mathrm{E}_{d}$ ) contour plot recorded with the Al-SDD - DBAIII preamplifier -4X amplifier (a) and the Au-SDD - DBAIV preamplifier (b). The structure of the event distribution in the contour plot reflects the time structure of the two bunches in the proton beam. The events from the two bunches are well separated in time only for deposited energies $E_{d}>10 \mathrm{MeV}$. For lower $\mathrm{E}_{d}$ values the two bunches overlap. Note that the grey scale is reversed and the maximum event concentration corresponds to a white colour.

Some peak structures with $>2000$ and $>4000$ events per bin for the Al-SDD and the Al-SDD can be recognised at $E_{d}$ values below $3 \mathrm{MeV}$. The peak at about $\left(t_{T o F}=42 \mathrm{~ns}, E_{d}<1 \mathrm{MeV}\right)$ is associated with a strong flash of $\gamma$-rays coming directly from the moderator and spallation target. This peak was used for synchronization of the ToF axis since it provides a more stable reference than the proton signal from the accelerator. An identical peak is visible about $320 \mathrm{~ns}$ later and is due to the second proton bunch. A broader peak structure is also visible at longer ToF $(\approx 800 \mathrm{~ns})$. The same peaks are visible in the ToF spectra shown in figure 6. One can see that the widths of the two $\gamma$-ray peaks are comparable to those of the proton signal peaks shown for comparison. It has to be stressed that the fine time structure of the falling edge of the two main peaks are appreciably different. The bump at around $480 \mathrm{~ns}$ is the signal relative to $\mathrm{n}^{12} \mathrm{C}$ elastic scattering in the region $\mathrm{E}_{n}=3-4 \mathrm{MeV}$ (for neutrons belonging to the first burst), where the elastic cross section shows a broad peak. This is confirmed by the presence of a second broad peak at $800 \mathrm{~ns}$, that is related to neutron of the same energy but belonging to the second burts. Indeed the time difference between the bump and the peak is about 320 ns, i.e. the proton bunches time distance, shown in figure 6.

Figure 7 shows the $E_{d}$ spectrum obtained by projecting the events in figure 5 on the vertical axis. Both spectra feature a peak at low energies $\left(E_{d} \approx 1 \mathrm{MeV}\right)$ and a long tail. In the range $2 \mathrm{MeV}<E_{d}<25 \mathrm{MeV}$ the two spectra are very similar. This appears to be the most promising range for use of the SDD as a neutron beam monitor and is further analysed below.

\section{Biparametric analysis}

The blue and red lines in figure 5 provide a guide for understanding the observed event distribution in $\left(t_{T o F}, E_{d}\right)$ space. The blue line is the maximum energy deposited by ${ }^{12} \mathrm{C}(\mathrm{n}, \alpha){ }^{9} \mathrm{Be}$ reactions of 


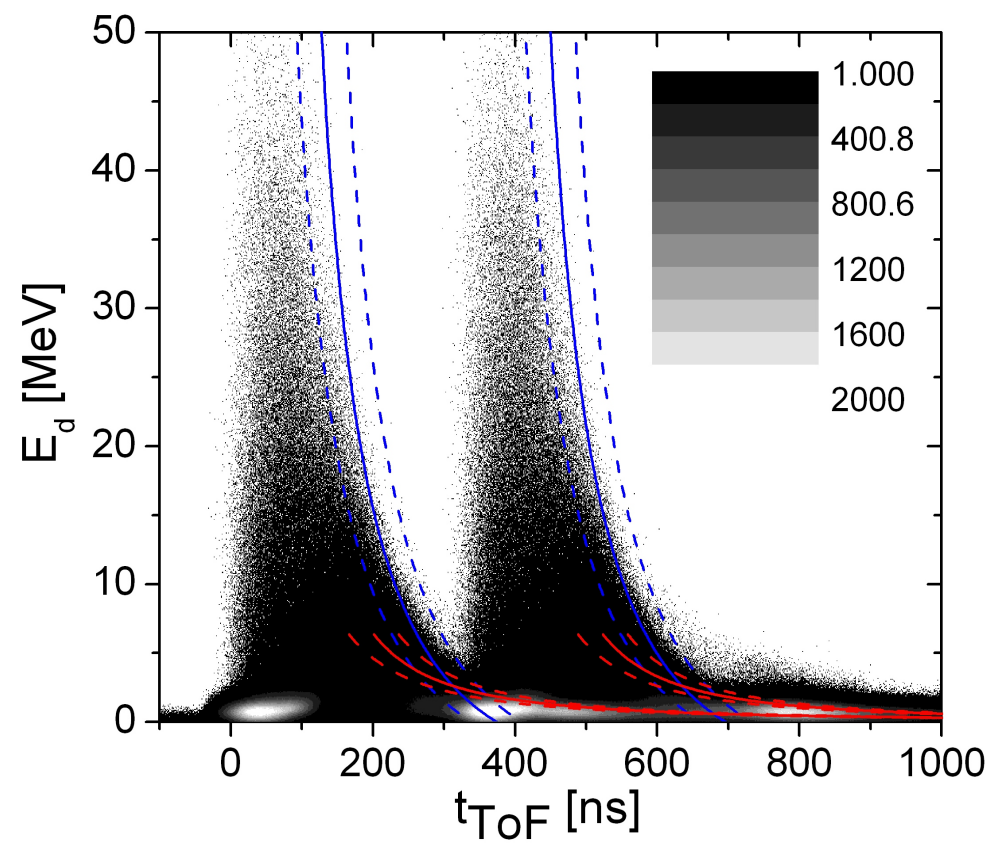

(a)

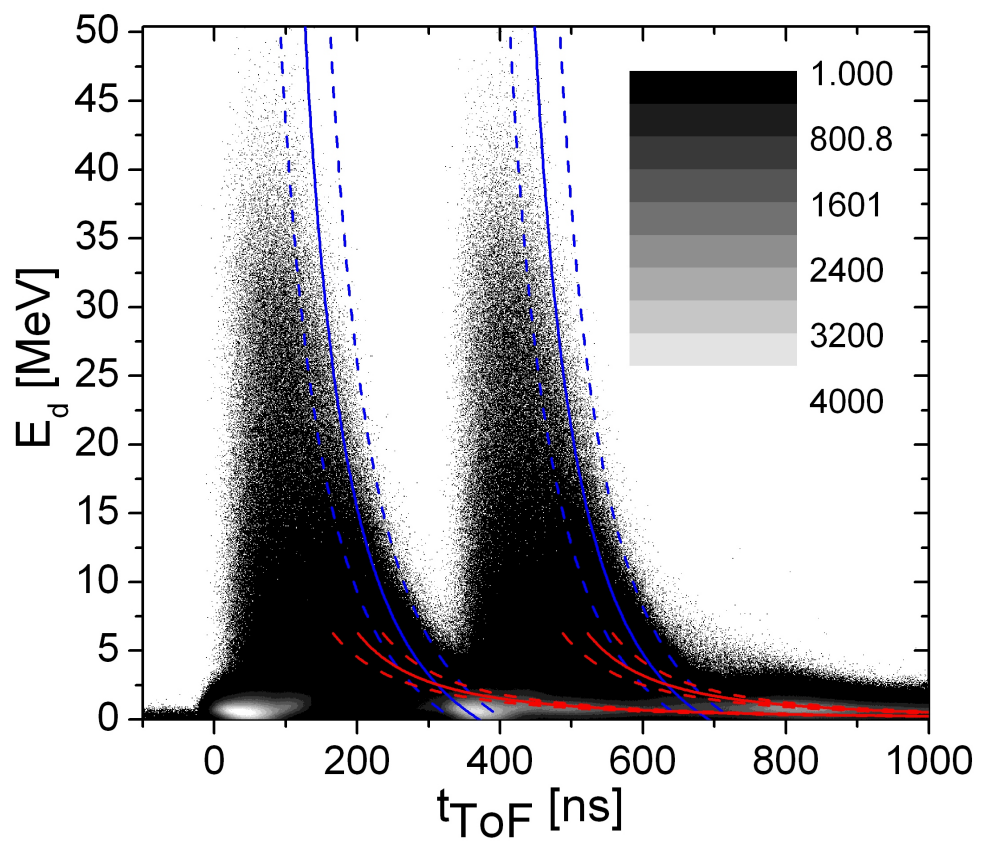

(b)

Figure 5. Contour plot of the event density in the $\left(\mathrm{t}_{T o F}, \mathrm{E}_{d}\right)$ plane for the Au-SDD (a) and the Al-SDD (b). The total number of events in the plot is $4.39 \times 10^{7}$ (Al-SDD) and $1.73 \times 10^{7}$ (Au-SDD). The bin width is 1 ns in $\mathrm{t}_{T o F}$ and 0.075 and $0.050 \mathrm{MeV}$ in $\mathrm{E}_{d}$ for the Au-SDD and $\mathrm{Al}$ SDD respectively. The integrated proton beam current was $4.401 \mathrm{mAh}$ corresponding to a data collection time of $32 \mathrm{~h}$. The blue and red lines are the maximum deposited energy for the case of ${ }^{12} \mathrm{C}(\mathrm{n}, \alpha){ }^{9} \mathrm{Be}$ and elastic ${ }^{12} \mathrm{C}\left(\mathrm{n}, \mathrm{n}^{\prime}\right){ }^{12} \mathrm{C}$ scattering reactions, respectively. The dashed lines are the same as the full lines but with a shift of $\pm 35 \mathrm{~ns}$ in the $\mathrm{t}_{T o F}$ value. 


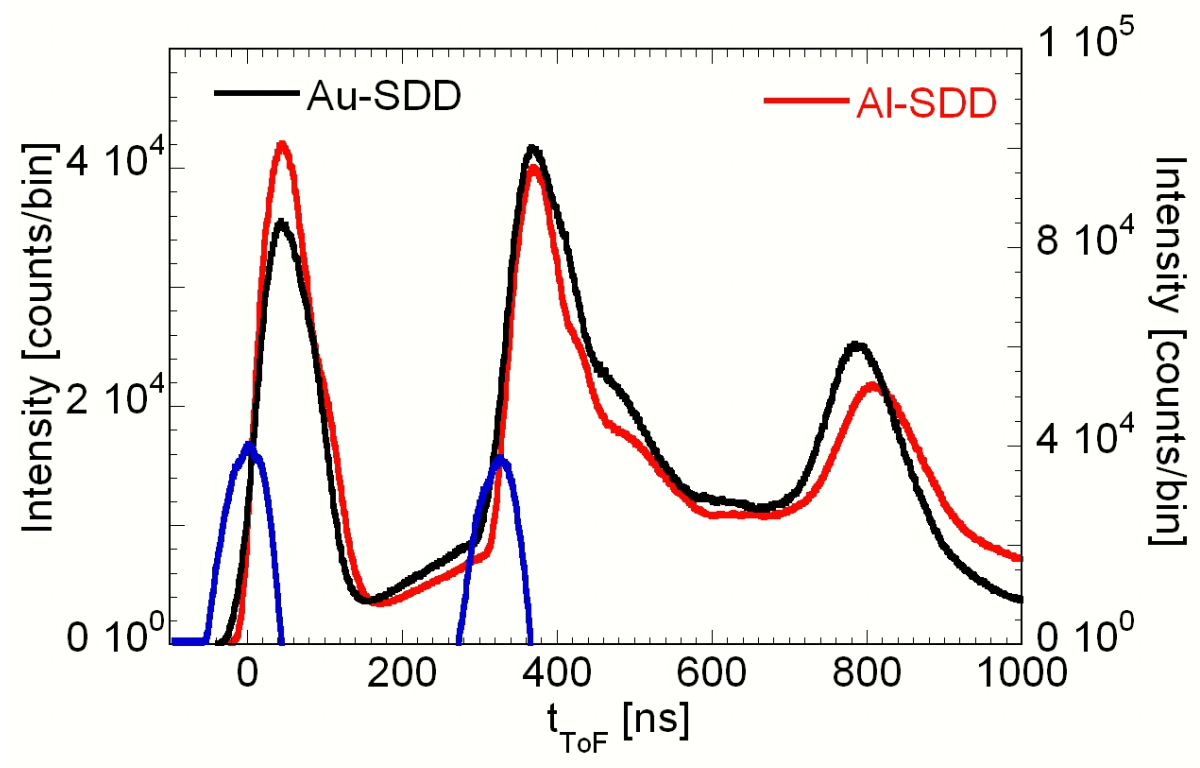

Figure 6. Time of flight spectrum for the two detector-amplification combinations used. The black line represents the spectrum for the Au-SDD - DBAIV preamplifier obtained by projecting the data of figure 5 on the horizontal axis. The red line is the same but for Al-SDD - DBAIII preamplifier - 4X amplifier combination. Also shown in blue is the proton pulse signal from the accelerator in arbitrary units.

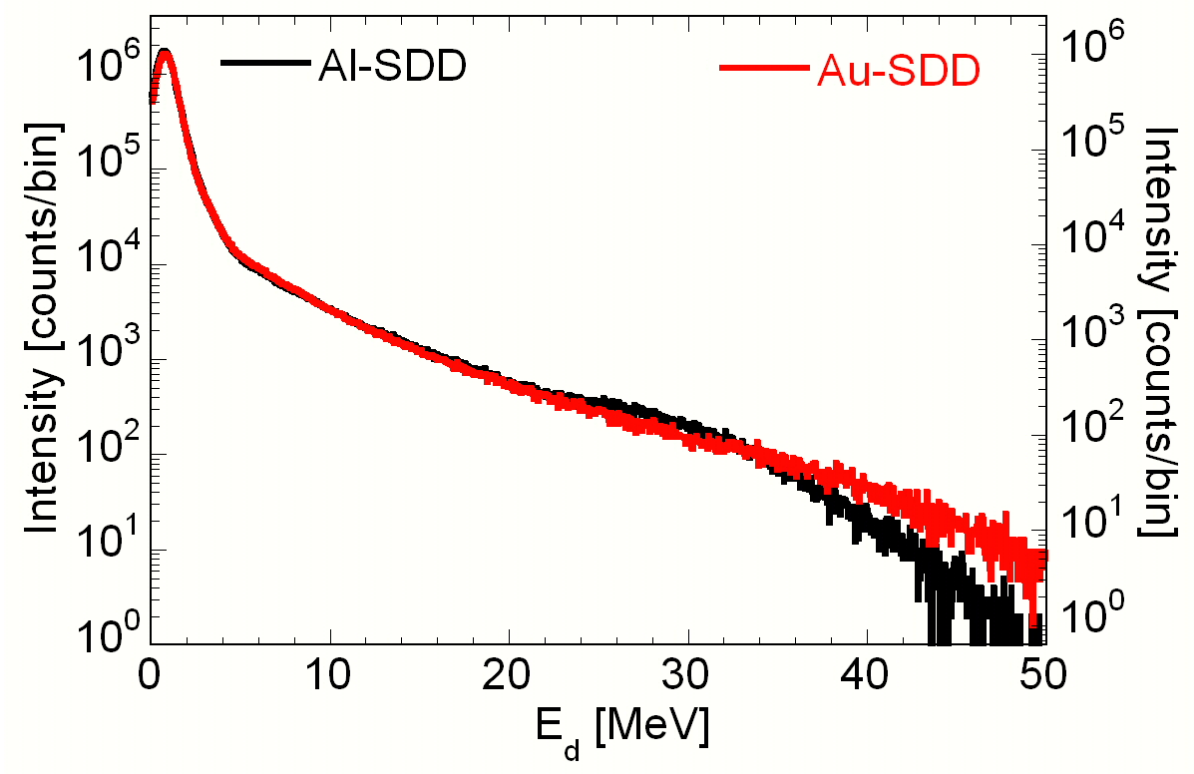

Figure 7. Spectrum of the deposited energy for the two detector-amplification combinations used. The black line represents the $\mathrm{E}_{d}$ spectrum for the Au-SDD - DBAIV preamplifier obtained by projecting the data of figure 5 on the vertical axis. The red line is the same but for Al-SDD - DBAIII preamplifier - 4X amplifier combination. The $\mathrm{E}_{d}$ bin width is the same as in figure 6 . The vertical scales are adjusted to provide a good match between the two traces in the range $5 \mathrm{MeV}<\mathrm{E}_{d} \quad<25 \mathrm{MeV}$. 


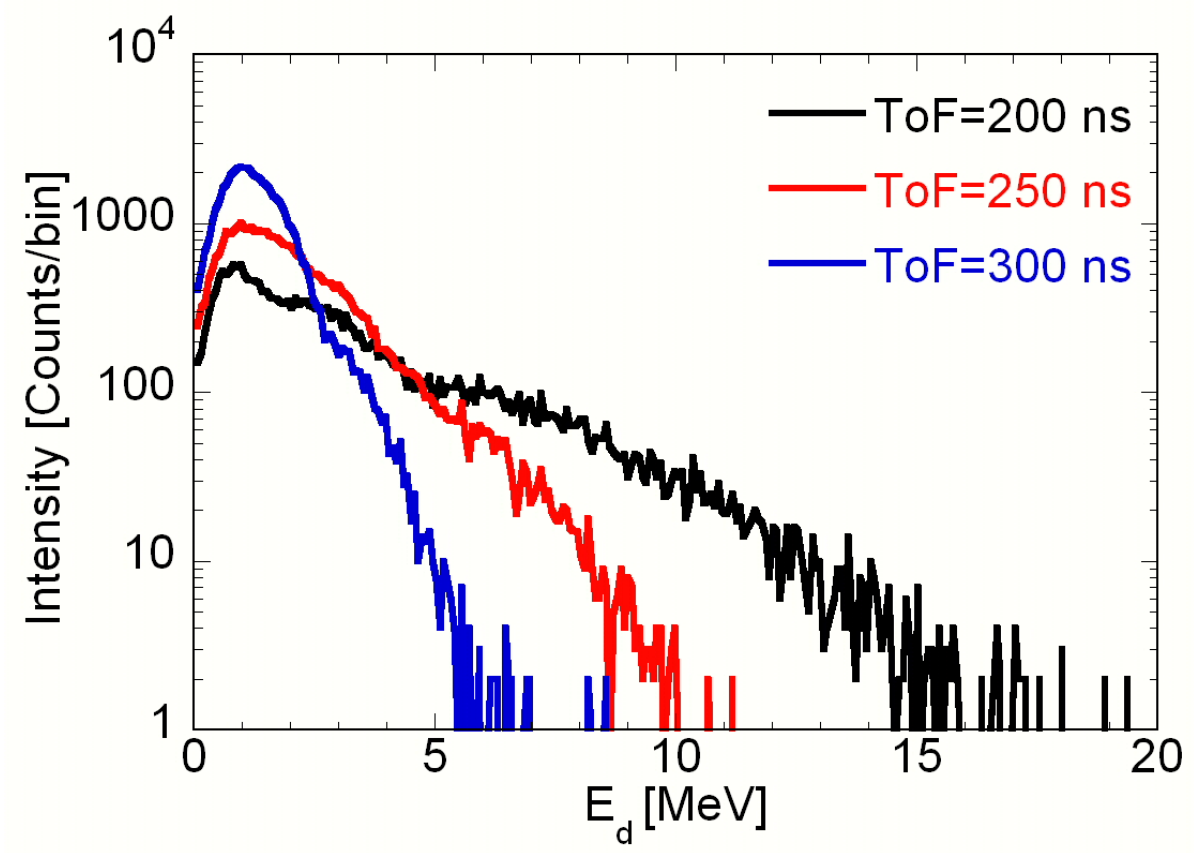

Figure 8. $\mathrm{E}_{d}$ spectrum for the Au-SDD for the ToF values reported in the legend. The ToF bin width is $5 \mathrm{~ns}$. The $\mathrm{E}_{d}$ bin width is $0.075 \mathrm{MeV}$.

neutrons having the energy corresponding to the ToF value; i.e.

$$
E_{n}=\left(\frac{1}{\sqrt{1-\left(\frac{L}{t_{T o F}}\right)^{2}}}-1\right) \cdot m c^{2} \quad E_{d}=E_{n}+Q, \quad Q=m_{n}+m_{C}-m_{B e}-m_{\alpha}
$$

where $E_{n}, E_{d}$ and the neutron mass are in energy units, $c$ is the speed of light, $L=12.5 \mathrm{~m}$ is the neutron flight distance and $Q=5.7 \mathrm{MeV}$ is the reaction negative $\mathrm{Q}$-value calculated from the atomic masses of the nuclei. Other break up reactions such as ${ }^{12} \mathrm{C}\left(\mathrm{n}, \mathrm{n}^{\prime}\right) 3 \alpha$ ave a higher threshold and provide events that should fall below the blue curve. This is indeed what is observed for short flight times provided one takes into account the $\pm 35 \mathrm{~ns}$ time spread of the proton pulse illustrated by the dashed blue lines. For flight times longer than $\approx 300 \mathrm{~ns}$, where $E_{n}$ is low and ${ }^{12} \mathrm{C}\left(\mathrm{n}, \mathrm{n}^{\prime}\right) 3 \alpha$ is below threshold, there is a significant amount of events (best seen in the second bunch of events i.e. at $t_{T o F}>700 \mathrm{~ns}$ ). This is where a broader peak structure is observed in figure 5. A possible explanation for these events is that they are due to neutron elastic scattering off carbon. The recoiling carbon nuclei have a maximum energy (head on collisions) amounting to $28 \%$ of the incoming neutron energy. The corresponding $E_{d}$-ToF curve is shown in red in figure 5. The ToF range where elastic scattering seems to be the main interaction process is $t_{T o F}=380-580 \mathrm{~ns}$. The corresponding neutron energy interval is $2.4<E_{n}<5.7 \mathrm{MeV}$.

Further insight in the data can be gained by slicing the $2 \mathrm{D}$ data plot along lines of constant ToF or constant $E_{d}$. In figure 8, the $E_{d}$ spectrum for different neutron ToF is plotted. The $t_{T o F}$ bin width was chosen equal to $5 \mathrm{~ns}$ in order to achieve sufficient statistics. The ToF values correspond 


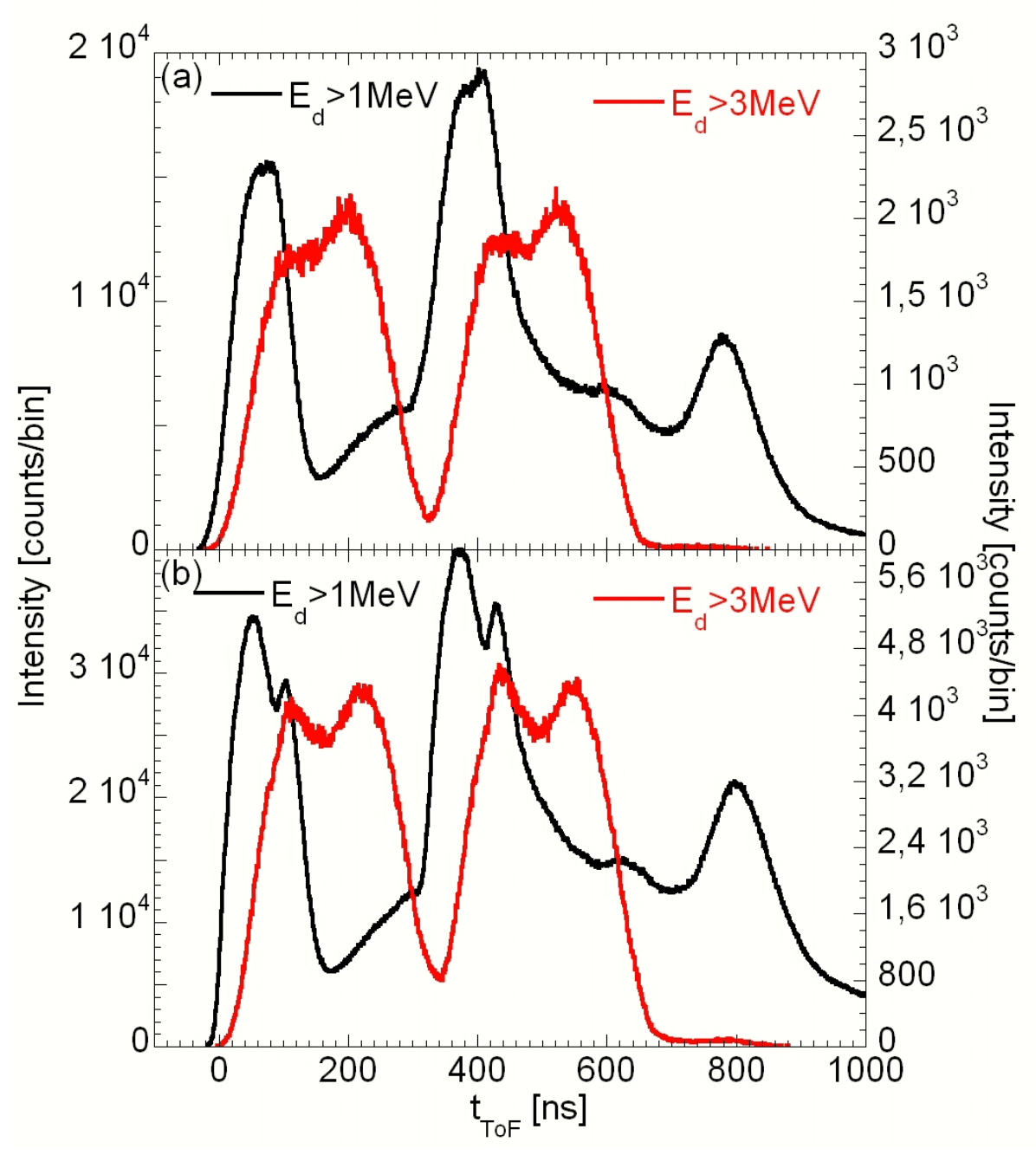

Figure 9. ToF spectrum for the Au-SDD (a) and for the Al-SDD for the $\mathrm{E}_{d}$ intervals reported in the legend. The ToF bin width is $1 \mathrm{~ns}$.

to an (average) neutron energy equal to $E_{n}=21.1 \mathrm{MeV}\left(t_{T o F}=200 \mathrm{~ns}\right), E_{n}=13.3 \mathrm{MeV}$ ( $t_{T o F}=250 \mathrm{~ns}$ ), $E_{n}=9.2 \mathrm{MeV}\left(t_{T o F}=300 \mathrm{~ns}\right)$. There is a clear correlation between average $E_{n}$ and maximum $E_{d}$. A more quantitative analysis of the spectra would require knowledge of the SDD response to monoenergetic neutrons folded with the relatively broad time distribution of the protons.

ToF spectra for the Au-SDD (a) and Al-SDD (b) for two different thresholds are reported in figure 9. The black line represent the ToF spectrum for events with $\mathrm{E}_{d}>1 \mathrm{MeV}$, while the red line $\mathrm{E}_{d}>3 \mathrm{MeV}$. The first one is mainly due to both $\gamma$-rays and low energy neutrons interacting in the detector, thus is very similar to spectra shown in figure 6 . The spectra in red are very different because they are due to high energy neutrons that interact in diamond mainly via ${ }^{12} \mathrm{C}(\mathrm{n}, \alpha){ }^{9} \mathrm{Be}$ reaction. Because the energy threshold of this reaction is $6.17 \mathrm{MeV}$ the two bunches are well separated. Concerning the single bunches, we do not have a clear explanation for the double structure for the two peaks for $\mathrm{E}_{d}>3 \mathrm{MeV}$. This is due to the way neutrons at about $\mathrm{E}_{n}=17-20 \mathrm{MeV}$ interact into the diamond. Above this energy the neutron interaction cross section is not completed 


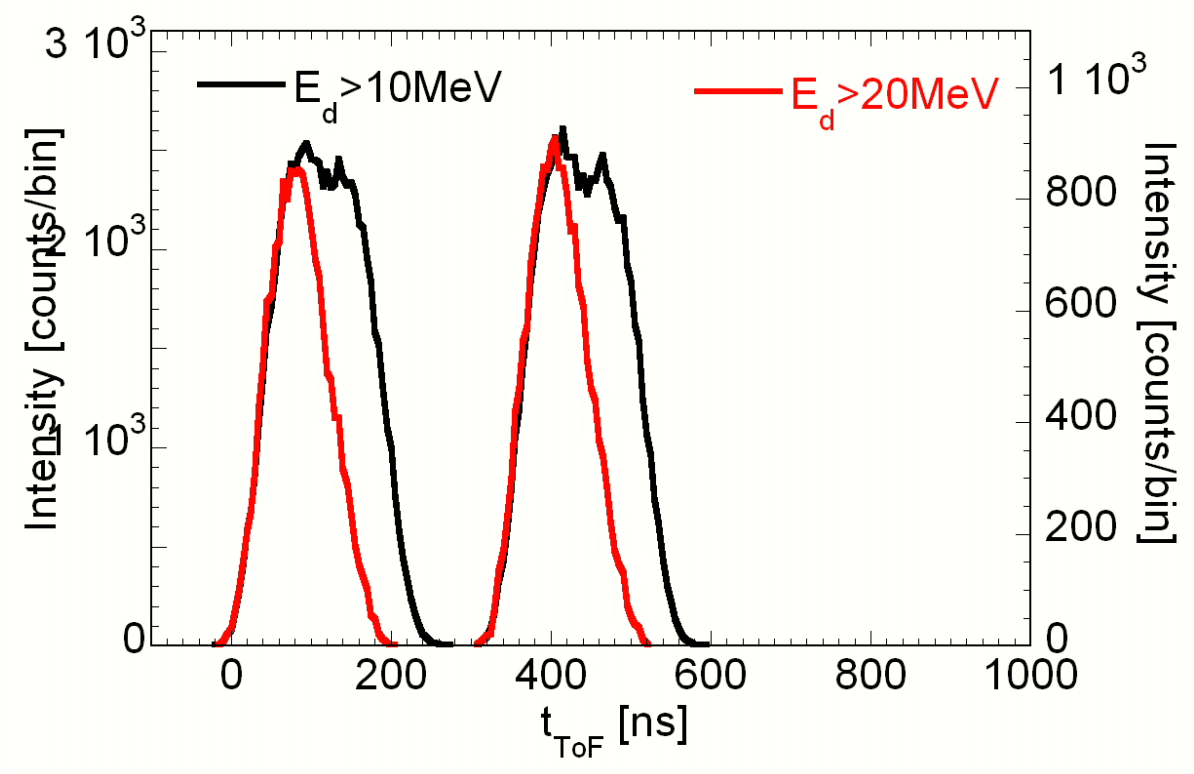

Figure 10. ToF spectrum for the Au-SDD for the $\mathrm{E}_{d}$ intervals reported in the legend. The ToF bin width is $5 \mathrm{~ns}$.

assessed. This is the subject of further measurements we plan to perform at the nTOF beamline at CERN, where beam characteristics and the beam line layout will allow for a better insight into the detector response. The slight difference between the spectral shape of the Au-SDD and Al-SDD in probably due to the $\mathrm{E}_{d}$ calibration uncertainty.

ToF spectra obtained for larger $E_{d}$ thresholds are shown in figure 10. Again a clear correlation between $t_{T o F}$ and $E_{d}$ is observed: the maximum $t_{T o F}$ is shorter for the higher energy. A neutron that deposits $10 \mathrm{MeV}$ in the SDD should have $E_{n}>15.7 \mathrm{MeV}$; that is $t_{T o F}<230 \pm 35 \mathrm{~ns}$. A neutron that deposits $20 \mathrm{MeV}$ should have $E_{n}>25.7 \mathrm{MeV}$, i.e. $t_{T o F}<182 \pm 35 \mathrm{~ns}$. The spectra in figure 10 are in qualitative agreement with the above $t_{T o F}$ limits. Again, for a quantitative analysis knowledge of the SDD response to monoenergetic neutrons is needed. Future work will include the improvement of the data reduction for optimal energy resolution before the SDD response function is determined at a suitable neutron source.

\section{Beam profile measurements}

The last measurement performed at the VESUVIO beamline with the Al-SDD coupled to the DBAIII was a horizontal scan of the detector position in order to determine the spatial profile of the neutron flux. Figure 11 represents the horizontal beam profile for different regions in the $\left(t_{T o F}, E_{d}\right)$ space. The number of events were normalized to 1 . Three regions are considered:

i) $E_{d}<5 \mathrm{MeV}$ and $t_{T o F}<75 \mathrm{~ns}$. Here the events are mainly due to photon interactions. The profile width (FWHM) is $W=45.5 \mathrm{~mm}$

ii) $E_{d}>5 \mathrm{MeV}, 200<t_{T o F}<250 \mathrm{~ns}$. The events in this range are mainly due to inelastic n-C reactions with intermediate $E_{n}$ values. The profile width (FWHM) is $W=37 \mathrm{~mm}$ 


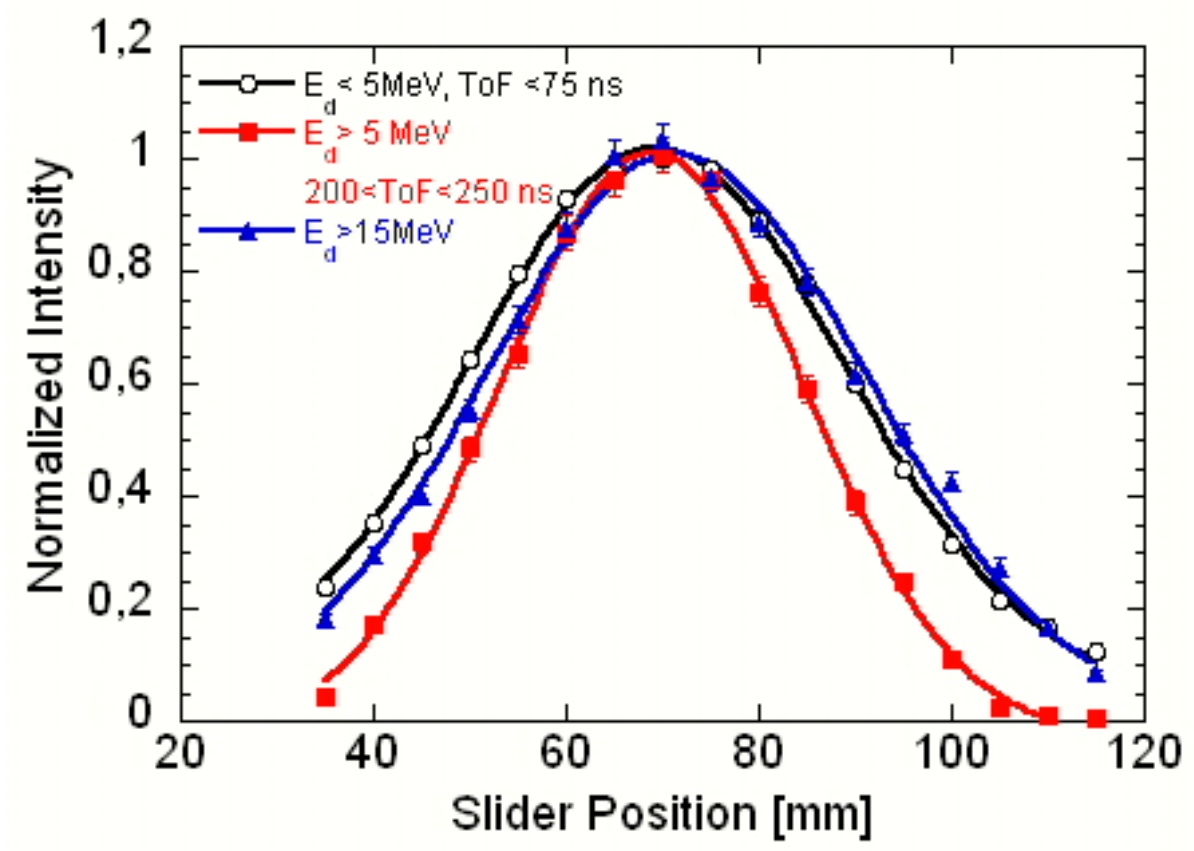

Figure 11. Horizontal beam profile obtained by selecting events with (black) $\mathrm{E}_{d}<5 \mathrm{MeV}$ and $\mathrm{t}_{T o F}<75$ ns, (red) $\mathrm{E}_{d}>5 \mathrm{MeV}, 200<\mathrm{t}_{T o F}<250 \mathrm{~ns}$, and $\mathrm{E}_{d}>15 \mathrm{MeV}$ (blue). Each point corresponds to a 15 minute long run. The integrated current was typically $45 \mu \mathrm{Ah}$ in each point. The beam profiles were normalized to 1 in order to better underline the shape. The lines are a Gaussian fit to the data.

iii) $E_{d}>15 \mathrm{MeV}$. This region includes events due to high energy neutrons. The profile width (FWHM) is $W=46.6 \mathrm{~mm}$.

The differences in profile width are well outside the uncertainties in the measurement.

Gamma and high energy neutrons (black and blue curves, region i) and iii)) have a broader profile than intermediate energy neutrons (red curve, region ii)). This is not surprising, given that the VESUVIO collimation was optimized for thermal and epithermal neutrons, and not for gamma rays or very high energy neutrons. It would be interesting to compare the results with model simulations of the neutron and $\gamma$-ray collimation along the VESUVIO beam line. This simulation work will be addressed in the future. Once the SDD response to monoenergetic neutrons becomes available it should be possible to unfold the biparametric spectrum and determine the beam profile as function of neutron energy. This will be especially interesting on the ChipIr beam line where different modes of operation will be available providing a range of beam profiles. The measurement of the beam profile at different neutron energies will be a powerful benchmark for the neutron transport simulations on which the ChipIr beam line design is based.

\section{Discussion on the expected neutron spectra}

The results obtained on the VESUVIO beamline show the SDD potential and limitations for neutron spectrometry at ISIS in the $\mathrm{MeV}$ region. Although the time structure of the ISIS proton bunch complicates and partly destroys the relation between ToF and neutron energy, figures 9-10 show 
that there is quantitative ToF information that can be used e.g. to benchmark neutron spectrum predictions. A more quantitative interpretation to the data would require knowledge of the detector efficiency and response to monoenergetic neutrons. We plan to determine the SDD response experimentally at the nTOF beamline of CERN where the proton bunch width is $5 \mathrm{~ns}$ (FWHM) and the neutron energy is precisely determined by the ToF. In order to determine the SDD absolute efficiency cross-calibration with an absolute reference (e.g. a Fission Chamber) is required.

As the neutron energy is raised to tens of $\mathrm{MeV}$ we expect partial energy deposition to occur. This can occur when the secondary charge particle, such as an $\alpha$-particle from the ${ }^{12} \mathrm{C}(\mathrm{n}, \alpha){ }^{9} \mathrm{Be}$ reaction, has a range longer than the detector thickness. As an example, a $50 \mathrm{MeV} \alpha$ particle has a range which is equal to $500 \mu \mathrm{m}$. This corresponds to a neutron energy greater than $80 \mathrm{MeV}$. In this case we have that only part of the neutron energy is deposited in the detector. Again, this does not affect the detector efficiency provided the energy deposited in the SDD exceeds the $\mathrm{E}_{d}$ threshold adopted in the measurements.

\section{Conclusions}

The performance of a single crystal diamond $\left(4.7 \times 4.7 \times 0.5 \mathrm{~mm}^{3}\right.$ active volume) detector was tested in the ISIS pulsed neutron beam using biparametric (time of flight and pulse height) data acquisition. Three characteristic regions in the biparametric spectra were observed: i) low pulse height events with very short time of flight induced by $\gamma$-rays; ii) low pulse height events at longer flight times (i.e. neutron energies $E_{n}>3.5-6 \mathrm{MeV}$ ), possibly due to neutron elastic scattering off ${ }^{12} \mathrm{C}$; iii) events with large pulse height and flight times corresponding to $E_{n}>6 \mathrm{MeV}$ mainly due to inelastic reactions such as $\left.{ }^{12} \mathrm{C}(\mathrm{n}, \alpha)\right)^{9} \mathrm{Be}$ and ${ }^{12} \mathrm{C}\left(\mathrm{n}, \mathrm{n}^{\prime}\right) 3 \alpha$. The SDD is a promising detector in view of applications to the ChipIr neutron beam line for fast neutron irradiation of electronic components at ISIS. Applications to be further investigated with the help of response function measurements and neutron transport simulations include beam profile measurements, beam intensity monitoring and broadband measurements of the neutron energy spectrum.

\section{Acknowledgments}

This work was supported within the CNR-STFC Agreement concerning collaboration in scientific research at the spallation neutron source ISIS. The financial support of the Consiglio Nazionale delle Ricerche in this research is hereby acknowledged.

\section{References}

[1] E. Normand, Single event upset at ground level, IEEE Trans. Nucl. Sci. 43 (1996) 2742.

[2] P.E. Dodd, M.R. Shaneyfelt, J.R. Schwank and G.L. Hash, Neutron-induced soft errors, latchup, and comparison of SER test methods for SRAM technologies, in Proceedings of the International Electron Device Meeting, Albuquerque U.S.A. (2002), pg. 333.

[3] Los Alamos Neutron Science Center webpage, http://lansce.lanl.gov/.

[4] TRIUMF: Canada's national laboratory for particle and nuclear physics webpage, http://www.triumf.ca/welcome/index.html.

[5] TSL: The Svedberg Laboratory webpage, http://www.tsl.uu.se/. 
[6] ISIS pulsed neutron and muon source at the Rutherford Appleton Laboratory webpage, http://www.isis.stfc.ac.uk/.

[7] V.P. Eismont, A.V. Prokofiev and A.N. Smirnov, Thin film breakdown counter and their applications (review), Radiat. Meas. 25 (1995) 151.

[8] A.N. Smirnov et al., Characterisation of the high-energy neutron field at the ISIS-VESUVIO facility by means of thin-film breakdown counters, technical report RAL-TR-2010-024, Rutherford Appleton Laboratory, Chilton U.K. (2010) [ISSN:1358-6254].

[9] M. Angelone et al., Single crystal artificial diamond detectors for VUV and soft X-rays measurements on JET thermonuclear fusion plasma, Nucl. Instrum. Meth. A 623 (2010) 726.

[10] P. Dong et al., Beam condition monitoring with diamonds at CDF, IEEE Trans. Nucl. Sci. 55 (2008) 328.

[11] D. Lattanzi et al., Single crystal CVD diamonds as neutron detectors at JET, Fus. Eng. Des. 842009 1156.

[12] A. Zimbal, L. Giacomelli, R. Nolte and H. Schumacher, Characterization of monoenergetic neutron reference fields with a high resolution diamond detector, Radiat. Measur. 45 (2010) 1313.

[13] M. Angelone et al., Radiation hardness of a polycrystalline chemical-vapor-deposited diamond detector irradiated with 14 MeV neutrons, Rev. Sci. Instrum. 77 (2006) 023505.

[14] Diamond Detector Ltd., BCMS-SCD464650A - BCM, U.K. (2012).

[15] M. Pillon et al., Experimental response functions of a single-crystal diamond detector for 5-20.5 MeV neutrons, Nucl. Instrum. Meth. A 640 (2011) 185.

[16] M. Pillon, M. Angelone and A.V. Krasilnikov, 14 MeV neutron spectra measurements with 4\% energy resolution using a type IIa diamond detector, Nucl. Instrum. Meth. B 101 (1995) 473.

[17] G.J. Schmid et al., A neutron sensor based on single crystal CVD diamond, Nucl. Instrum. Meth. A 527 (2004) 554.

[18] A. Pietropaolo et al., Single-crystal diamond detector for time-resolved measurements of a pulsed fast-neutron beam, Europhys. Lett. 92 (2010) 68003.

[19] L. Giacomelli et al., Diamond detectors for fast neutron irradiation experiments, Nucl. Phys. Proc. Suppl. B 215 (2011) 242.

[20] A. Pietropaolo et al., Fission diamond detectors for fast-neutron ToF spectroscopy, Europhys. Lett. 94 (2011) 62001.

[21] M. Rebai et al., Fission diamond detector tests at the ISIS spallation neutron source, Nucl. Phys. Proc. Suppl. B 215 (2011) 313.

[22] L. Giacomelli et al., Diamond detector for high rate monitors of fast neutron beams, Funfi Proceeding Conference, in press, Varenna Italy (2011).

[23] CAEN - Costruzioni Apparecchiature Elettroniche Nucleari S.p.A., DT5751 2/4 channel 10 bit 2/1 GS/s digitizer webpage, http://www.caen.it/csite/CaenProd.jsp?parent=14\&idmod=632.

[24] C. Andreani et al., CdZnTe detector for deep inelastic neutron scattering on the VESUVIO spectrometer, Appl. Phys. A 78 (2004) 903.

[25] P. Moritz, Broadband preamplifiers for fast particle detectors, GSI — Gesellschaft für Schwerionenforschung mbH, Planckstr. 1 D-64291 Darmstadt Germany.

[26] Python programming language - official website, http://www.python.org/. 